\title{
Attack of the cyberspider
}

The quest for quantum computing seems a little like that for fusion energy - the necessary technology always seems another decade away, receding into the future almost as fast as we chase it. So far, no one has built a device that has more than about 10 qubits, or carried out any computation that would be impossible on a classical device. The potential for new information devices exploiting quantum physics is surely real enough - as quantum random-number generators and systems for quantum key distribution have already hit the market - but quantum computation, despite unambiguous progress, still seems a reality that we are approaching asymptotically, to be realized (according to current estimates) around 2020 or so.

Even so, it's clear that computation will be very different well before 2020, and even without quantum technology. The more immediate transforming technology is emerging from techniques for controlling and manipulating single molecules, especially information-rich biomolecules, and for constructing what is coming to be called 'molecular cybernetics'.

Double-stranded DNA may be the basis of life, but single-stranded DNA may turn out to be more promising for computing. In impressive work over the past few years, for example, Milan Stojanovich of Columbia University and colleagues have used it to design a number of simple logical gates based on chemical activity. They base their gates on a nucleic acid enzyme - a deoxyribozyme - which catalyses certain DNA reactions. By attaching to this enzyme so-called stem loops - short single-stranded oligonucleotides that bind and inhibit the enzyme activity - they can make this activity sensitive to the presence or absence of further strands of DNA that can disrupt the effects of the stem loops.

This is a little complicated, but the result is a chemical logic in which the presence or absence of specific DNA strands represents logical inputs -1 or $0-$ and the enzyme being active or inactive (reflected in its ability to cleave a certain test oligonucleotide) gives the output of a gate, also 1 or 0 , acting on those inputs. With fluorescent markers to detect such activity, the readout can show up directly in colours.

Using various different stem loops, these researchers have managed to design a

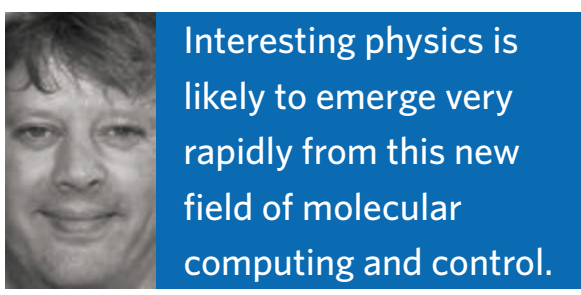

variety of logic gates including NOT, AND, OR and so on, and, by combining them, to devise automata capable of playing simple games, such as Tic-Tac-Toe (or 'noughts and crosses', as it's also known). The most recent such implementation uses more than 100 basic logic gates distributed among a set of fluid wells making up the game board. In their set-up, the automaton always goes first, choosing the middle well, after which a human player can respond by choosing any of the other eight wells. The chemical system of gates then calculates subsequent moves, and fluorescent markers make the wells turn red (for the automata) and green (for the human). The chemical system has yet to be defeated.

This isn't meant, of course, to be anything other than a proof of principle, and Stojanovich and colleagues point out that this kind of computation will never compete with solid-state devices in terms of speed. Indeed, the Tic-TacToe automaton takes about 30 minutes to make each of its moves, as the underlying DNA chemistry is quite slow. But computation isn't only about speed, even if this is what we usually emphasize in our thinking about it. The potentially revolutionary aspect of this technology is that the computation works in solution, and could, for example, be used to carry out information processing in biological fluids.

Hence, it is easy to imagine future devices carrying out tasks considerably more complex than Tic-Tac-Toe, and doing so autonomously within living cells. There is clearly potential for the engineering of molecular control systems able to detect specific DNA sequences, for example, and to release specific drugs or molecules in response. Just as computation has spread rapidly into every corner of engineering control, we can expect the same kind of transformation of biology and medicine into fields dominated by control based on flexible chemical computation, and an intelligent and active chemistry that can gather molecular information and calculate delicate actions based on it.

In this regard, one of the most exciting recent developments is the creation, also by Stojanovich and colleagues, of molecular 'spiders' - biomolecular systems with 'legs' made of single-stranded DNA segments having lengths of the order of $10 \mathrm{~nm}$. These spiders can move over a surface covered with single-stranded DNA segments that are complementary to its legs, as they repeatedly bind, dissociate and bind again. The movement of such spiders in large numbers can be controlled by engineering the properties and geometry of the surface, as well as by the physical conditions influencing the statistics of the binding process.

As a result, it's clear that interesting physics is likely to emerge very rapidly from this new field of molecular computing and control, and some physicists have already become actively involved. In recent work, Tibor Antal and Paul Krapivsky have noted that there's an interesting feedback between these spiders and their environment. When a spider leg binds to a site, it typically breaks a bond within the oligonucleotide at that site; hence, a spider's first visit to a site alters that location in an irreversible way, and on future visits, the legs don't bind so strongly. Hence, the terrain a spider sees and its physical influence on a spider varies with the spider's activity, and its diffusion is significantly more complicated than that of a passive particle.

Here one has diffusion with a memory effect, and the proper description of such motion, as Antal and Krapivsky point out, demands the solution of nonMarkovian transport models, which they have only begun to explore.

The possibilities for controlling spiders and other novelties will only become richer with incredible objects like the DNA box reported last month (Nature 459, 73-76; 2009). Using a technique known as DNA origami, Ebbe S. Anderson and colleagues were able to make a $3 \mathrm{D}$ box a few tens of nanometres on each side with a lid that can be opened by presenting certain DNA keys.

Our future, it seems, may well be written in DNA, just not of the same form as our biological past.

MARK BUCHANAN 